\title{
LAMINA PAPYRACEA DEHISCENCE: CAUSE FOR RECURRENT PRESEPTAL CELLULITIS, ORBITAL CELLULITIS AND ORBITAL ABSCESS IN A CHILD
}

\author{
Ayşe Tekin Yılmaz ${ }^{1}$, Yonca Anık ${ }^{2}$ Emin Sami Arısoy ${ }^{1}$ \\ Kocaeli University Faculty of Medicine \\ ${ }^{1}$ Department of Paediatric Health and Diseases, Kocaeli, Turkey \\ 2Department of Radiology, Kocaeli, Turkey
}

Introduction

Preseptal cellulitis, orbital cellulitis and orbital abscess are not frequent in children. In recurrent cases (extremely rare), the existence of congenital faults as well as primary immune system problems must be considered.

Case

A 4-year old girl was admitted with redness/swelling in/around the right eye (Picture 1). In the past, she had frequent upper respiratory tract infections, and was treated for right orbital cellulitis, orbital abscess and after right preseptal cellulitis. In physical examination, the right eye would not open; its motion could not be assessed. There were no other particularities. Laboratory tests were normal except for elevation of acute phase reactants. MR imaging showed ethmoid and maxillary sinusitis, oedema in right preseptal/orbital areas and appearance compatible with abscess on the right orbital inner wall. Intravenous ampicillin-sulbactam and clindamycin administration was started. On the third day of hospitalization, the orbital abscess was surgically discharged. There was no culture growth in the abscess sample. Immunological and sweat tests were normal. In MR imaging on the $14^{\text {th }}$ day, the occasionally defective appearance of bilateral lamina papyracea was compatible with lamina papyracea dehiscence. Sinusitis findings continued and focal destruction areas were formed on the right ethmoid cellular lateral wall (Picture 2). On the $17^{\text {th }}$ day, treatment was complete and patient was discharged with no problems. After 20 days, she came back with right preseptal cellulitis findings. Surgical correction of lamina papyracea dehiscence and the anatomic defect formed on the side wall of the ethmoid sinus was planned after antibiotic treatment.

\section{Conclusion}

In recurrent preseptal cellulitis, orbital cellulitis or orbital abscess, the presence of congenital or acquired anatomical faults must also be investigated and the possibility of lamina papyracea dehiscence must be kept in mind (Picture 3 ).

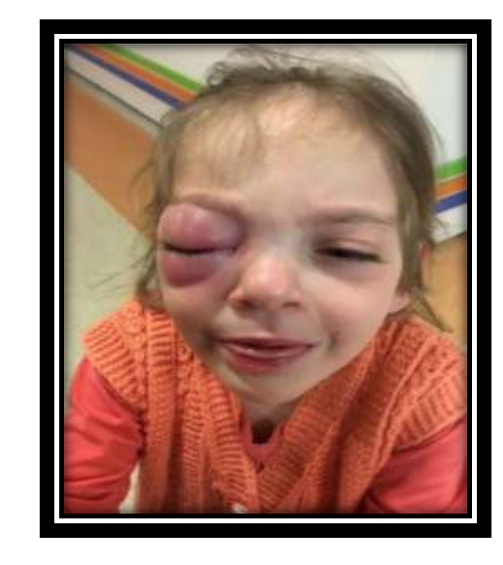

Picture 1: First admission

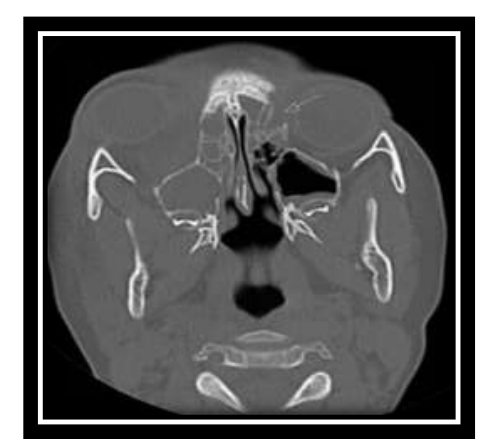

Picture 2: Radiologic image of lamina Papyracea dehiscence

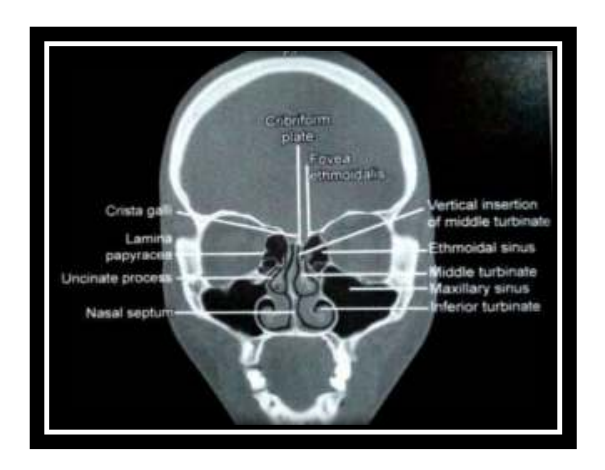

Picture 3: Anatomic location of lamina papyracea 